\title{
X-ray-laser interaction with matter and the role of multiphoton ionization: Free-electron-laser studies on neon and helium
}

\author{
A. A. Sorokin, ${ }^{1,2}$ M. Wellhöfer, ${ }^{3}$ S. V. Bobashev,${ }^{2}$ K. Tiedtke,${ }^{4}$ and M. Richter ${ }^{1}$ \\ ${ }^{1}$ Physikalisch-Technische Bundesanstalt, Abbestraße 2-12, D-10587 Berlin, Germany \\ ${ }^{2}$ Ioffe Physico-Technical Institute, Polytekhnicheskaya 26, 194021 St. Petersburg, Russia \\ ${ }^{3}$ Universität Hamburg, Luruper Chaussee 149, D-22761 Hamburg, Germany \\ ${ }^{4}$ Deutsches Elektronen-Synchrotron, Notkestraße 85, D-22603 Hamburg, Germany
}

(Received 6 December 2006; published 3 May 2007)

\begin{abstract}
At the soft-x-ray free-electron laser FLASH in Hamburg, we have studied multiphoton ionization on neon and helium by ion mass-to-charge spectroscopy. The experiments were performed in a focused beam at 42.8 and $38.4 \mathrm{eV}$ photon energy and irradiance levels up to $10^{14} \mathrm{~W} / \mathrm{cm}^{2}$. Direct, sequential, and resonant two-, three-, and four-photon excitations were investigated by quantitative measurements as a function of the absolute photon intensity. The atomic and ionic photoionization cross sections derived indicate a clear dominance of sequential compared to direct multiphoton processes.
\end{abstract}

DOI: 10.1103/PhysRevA.75.051402

PACS number(s): 32.80.Rm, 32.80.Fb, 42.50.Hz

Recent progress has been achieved in generating soft- $\mathrm{X}-$ ray pulses of high power by means of free-electron lasers (FELs) [1-4] and higher-harmonics generation technique [5]. In the near future, several large x-ray FEL facilities will be realized to study fast processes in materials and chemical reactions by ultrashort laser shots $[1,2,6]$. However, highly intense soft $\mathrm{x}$ rays (xuv) and vacuum-ultraviolet radiation cause nonlinear response of matter such as atomic multiphoton ionization [7-13] which has to be taken into consideration in any FEL experiment. In this context, we report on the strength of two-, three-, and four-photon multiple ionization obtained by quantitative measurements of ion time-offlight (TOF) spectroscopy at the new xuv Free-electronLASer in Hamburg (FLASH) [4]. In order to distinguish and compare different sequential and direct multiphoton excitation schemes, our experiments were performed on neon $(\mathrm{Ne})$ and helium (He) atoms at two different photon energies, namely 42.8 and $38.4 \mathrm{eV}$, i.e., just above and below the threshold for sequential two-photon double ionization of $\mathrm{Ne}$ via $\mathrm{Ne}^{+}$at $41.0 \mathrm{eV}$. Significant differences in the respective nonlinear dependences on photon intensity could be observed.

Figure 1 summarizes the multiphoton processes we discuss here. They refer to sequential [Fig. 1(d)] and direct [Fig. $1(\mathrm{~g})]$ two-photon processes, a combination of both, i.e., a four-photon excitation ending up in a triply charged ion [Fig. $1(\mathrm{e})]$, and three-photon double ionization via virtual and resonance states [Figs. 1(b) and 1(c)]. The investigations were performed at the microfocus beamline BL2 at FLASH with an experimental setup described in detail previously $[12,15,16]$. It consists of a calibrated online gas-monitor photodetector and a conventional ion TOF spectrometer. In order to avoid effects due to space charge and secondary ionization, the pressure of the target gas homogeneously filling the vacuum chamber was controlled below $2 \times 10^{-4} \mathrm{~Pa}$. The FEL radiation was distributed among subsequent photon pulses separated by $200 \mathrm{~ms}$ with up to $3 \times 10^{12}$ photons per pulse and a pulse duration of $\Delta t=(25 \pm 8)$ fs $[4,17]$. A focal spot size of $A=(5.0 \pm 0.7) \times 10^{-6} \mathrm{~cm}^{2}$ was realized by means of an ellipsoidal mirror [16]. Ions generated in the focus were extracted toward the TOF spectrometer by a static electric field parallel to the polarization vector of the $100 \%$ polarized photon beam. Along the photon beam, the TOF spectrometer had an acceptance length of $1 \mathrm{~mm}$ and could be moved $\pm 2 \mathrm{~cm}$ around the focus.

As a first result, Fig. 2(a) shows an ion spectrum taken at a photon energy of $\hbar \omega=(42.8 \pm 0.4) \mathrm{eV}$ in the focus of BL2. The spectrum results from an accumulation of about 1000 shots with a mean photon number per pulse of $N_{p h}$ $=(6.8 \pm 0.7) \times 10^{11}$ which yields a mean photon exposure of $H_{p h}=N_{p h} / A=(1.4 \pm 0.2) \times 10^{17}$ photons per $\mathrm{cm}^{2}$ and a mean

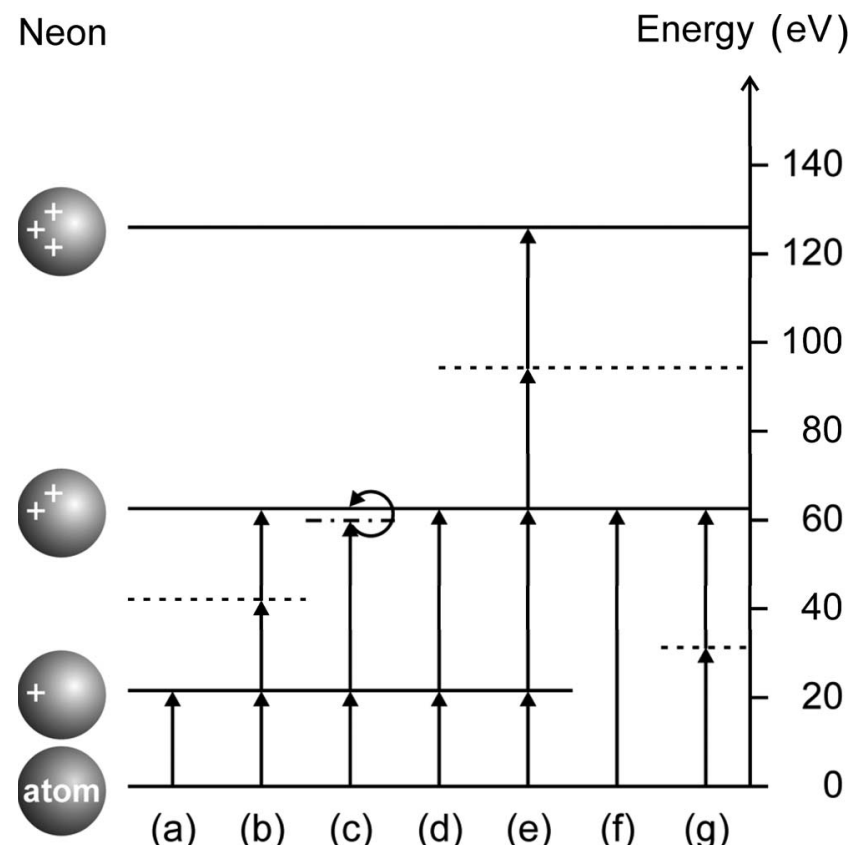

FIG. 1. Threshold energy diagram [14] and photoionization schemes for neon: (a) one-photon single ionization; (b) threephoton double ionization; (c) resonant three-photon double ionization; (d) sequential two-photon double ionization; (e) four-photon triple ionization; (f) one-photon double ionization; (g) direct twophoton double ionization. The dashed lines indicate virtual states. 


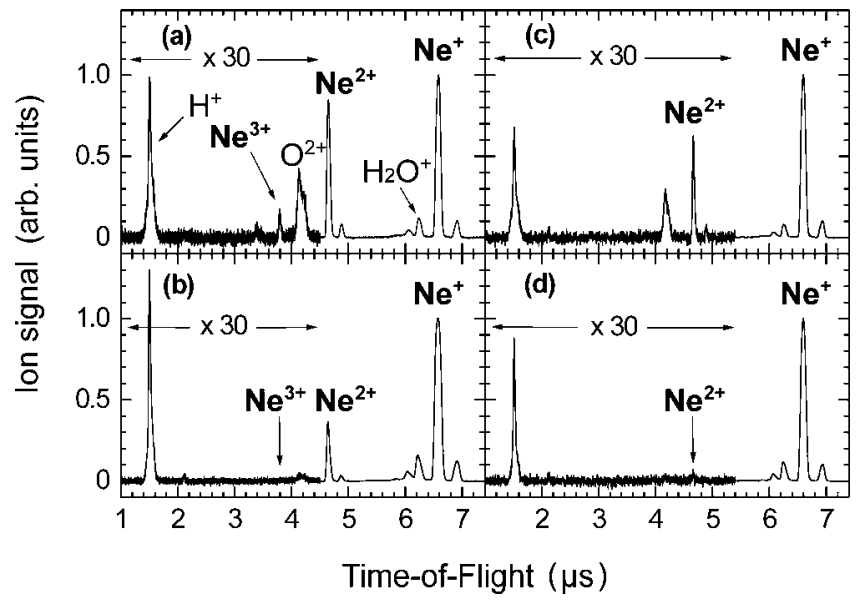

FIG. 2. Ion time-of-flight $(\mathrm{TOF}=\tau)$ spectra of neon taken at a photon energy of $42.8 \mathrm{eV}$ ( $\mathrm{a}$ and $\mathrm{b}$ ) and $38.4 \mathrm{eV}$ (c and d) in the focus of beamline BL2 at FLASH ( $a$ and $c$ ) and out of the focus (b and d) as a result of an accumulation of about 1000 shots with a mean photon number per pulse in the range from 6 to $8 \times 10^{11}$, respectively.

irradiance of $E=H_{p h}(\hbar \omega / \Delta t)=(3.7 \pm 1.3) 10^{13} \mathrm{~W} / \mathrm{cm}^{2}$. At such photon intensities, multiphoton processes are expected in the soft $\mathrm{x}$-ray range $[9,10,12]$. In fact, besides the dominating $\mathrm{Ne}^{+}$peak due to one-photon single ionization [Fig. 1(a) ] at a time of flight of $\tau=6.6 \mu$ s and various residual gas lines, a strong $\mathrm{Ne}^{2+}$ signal arises at $\tau=4.7 \mu \mathrm{s}$ and a weak $\mathrm{Ne}^{3+}$ signal at $\tau=3.8 \mu \mathrm{s}$. As demonstrated by Fig. 2(b), the $\mathrm{Ne}^{3+}$ signal completely disappears and the $\mathrm{Ne}^{2+}$ signal significantly decreases when moving the experimental setup by $1.5 \mathrm{~cm}$ out of focus where the spot size is three to four times larger and thus photon exposure and irradiance are correspondingly smaller [16]. Hence the $\mathrm{Ne}^{2+}$ and $\mathrm{Ne}^{3+}$ generation was not considerably affected by higher FEL harmonics at higher photon energy [18] because their contribution does not vary along the photon beam.

At a photon energy of $\hbar \omega=(42.8 \pm 0.4) \mathrm{eV}$, i.e., below the $\mathrm{Ne}$ threshold for one-photon double ionization at $62.6 \mathrm{eV}$ [Fig. 1(f)], the most probable explanation for $\mathrm{Ne}^{2+}$ generation seems to be, in accordance with recent results on molecular nitrogen [12], the sequential two-photon double ionization via $\mathrm{Ne}^{+}$[Fig. 1(d)] with a threshold energy of $21.6 \mathrm{eV}$ for the first step and $41.0 \mathrm{eV}$ for the second. The $\mathrm{Ne}^{3+}$ signal, on the other hand, is assumed to arise, in a further step, from the direct two-photon single photoionization of $\mathrm{Ne}^{2+}$ as an intermediate target, i.e., in total a fourphoton process [Fig. 1(e)]. In order to test these ideas, we have investigated the relative strength of $\mathrm{Ne}^{+}, \mathrm{Ne}^{2+}$, and $\mathrm{Ne}^{3+}$ generation in more detail by moving the setup along the photon beam and varying photon exposure $H_{p h}$ and irradiance $E$ (Fig. 3). In particular, the dependence of the photoionization rate $\dot{N}^{(n)}$ for a direct $n$-photon process on $E$ or $H_{p h}$ is approximated by [19]

$$
\dot{N}^{(n)}=N \sigma^{(n)}\left(\frac{E}{\hbar \omega}\right)^{n}=N \sigma^{(n)}\left(\frac{H_{p h}}{\Delta t}\right)^{n}
$$

with the initial number of targets $N$ and the $n$-photon ionization cross section $\sigma^{(n)}$. For the right part of Eq. (1), a rectan-

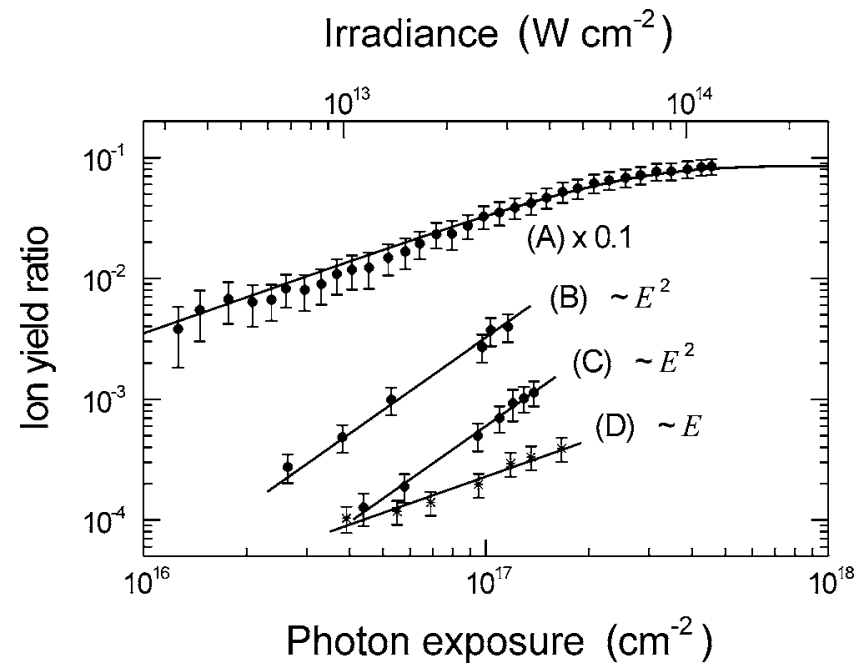

FIG. 3. Ion-yield ratios as a function of mean irradiance $(E)$ and photon exposure $\left(H_{p h}\right)$ : $\mathrm{Ne}^{2+}$ to $\mathrm{Ne}^{+}$ratio $(\mathrm{A}), \mathrm{Ne}^{3+}$ to $\mathrm{Ne}^{2+}$ ratio (C), $\mathrm{He}^{2+}$ to $\mathrm{He}^{+}$ratio (D) taken at $42.8 \mathrm{eV}$ photon energy and $\mathrm{Ne}^{2+}$ to $\mathrm{Ne}^{+}$ratio taken at $38.4 \mathrm{eV}$ photon energy (B). The data were corrected for the pulse-to-pulse statistics of the photon intensity and the relative detection efficiencies of the different ion species, respectively.

gular temporal distribution of the photon pulses is assumed. As a consequence, the $\mathrm{Ne}^{3+}$ to $\mathrm{Ne}^{2+}$ ion yield ratio should increase with the square of the photon exposure:

$$
\frac{N_{3+}^{(2)}}{N_{2+}}=\frac{\sigma_{\mathrm{Ne}^{2+} \rightarrow \mathrm{Ne}^{3+}}^{(2)}}{2}\left(\frac{E}{\hbar \omega}\right)^{2} \Delta t=\frac{\sigma_{\mathrm{Ne}^{2+} \rightarrow \mathrm{Ne}^{3+}}^{(2)}}{2} \frac{H_{p h}^{2}}{\Delta t}
$$

with the $n(=2)$-photon (single) ionization cross section of $\mathrm{Ne}^{2+} \sigma^{(n)}=\sigma_{\mathrm{Ne}^{2+} \rightarrow \mathrm{Ne}^{3+}}^{(2)}$. The mean number of intermediate $\mathrm{Ne}^{2+}$ targets during a single photon pulse has been approximated here by $N=\frac{1}{2} N_{2+}$ since at the beginning of the pulse, the number of intermediate $\mathrm{Ne}^{2+}$ targets is zero and at the end is equal to $N_{2+}$. Our measured $\mathrm{Ne}^{3+}$ to $\mathrm{Ne}^{2+}$ ratio is displayed by curve $\mathrm{C}$ in Fig. 3 together with a fit according to Eq. (2). The agreement between experimental data and model curve is almost perfect and yields a value of $\sigma_{\mathrm{Ne}^{2+} \rightarrow \mathrm{Ne}^{3+}}^{(2)}=(3.0 \pm 1.2) \times 10^{-51} \mathrm{~cm}^{4} \mathrm{~s}$ for this ionic direct two-photon ionization cross section as a fit parameter which is also listed in Table I (3). As an interesting consequence, studying sequential multiphoton ionization on atoms may yield fundamental information about photoionization of ions, too.

Within a pure sequential two-photon scheme for double ionization of $\mathrm{Ne}$ via $\mathrm{Ne}^{+}$[Fig. 1(d)] as an explanation for the strong $\mathrm{Ne}^{2+}$ signal in Fig. 2(a), also $\mathrm{Ne}^{+}$represents an intermediate target that arises from the first photoabsorption and vanishes with the second. From the general definition of the one-photon ionization cross sections $\sigma_{\mathrm{I}}=\sigma_{\mathrm{Ne} \rightarrow \mathrm{Ne}^{+}}^{(1)}$ for the first step and $\sigma_{\mathrm{II}}=\sigma_{\mathrm{Ne}^{+} \rightarrow \mathrm{Ne}^{2+}}^{(1)}$ for the second [Eq. (1) with $n=1$ ], a rate equation for the number $N_{+}$of intermediate $\mathrm{Ne}^{+}$targets generated per photon pulse within the interaction zone may be derived: 
TABLE I. Two-photon ionization cross sections of $\mathrm{Ne}, \mathrm{Ne}^{+}, \mathrm{Ne}^{2+}$, and $\mathrm{He}$.

\begin{tabular}{|c|c|c|c|c|}
\hline No. & Target & Photon energy (eV) & Two-photon process & Cross section $\left(\mathrm{cm}^{4} \mathrm{~s}\right)$ \\
\hline (1) & $\mathrm{Ne}$ & $(42.8 \pm 0.4)$ & $\begin{array}{l}\text { sequential two-photon } \\
\text { double ionization }\end{array}$ & $(7.2 \pm 2.5) \times 10^{-49 a}$ \\
\hline$(2)$ & $\mathrm{Ne}^{+}$ & $(38.4 \pm 0.4)$ & $\begin{array}{l}\text { resonant two-photon } \\
\text { single ionization }\end{array}$ & $(1.6 \pm 0.6) \times 10^{-50}$ \\
\hline (3) & $\mathrm{Ne}^{2+}$ & $(42.8 \pm 0.4)$ & $\begin{array}{l}\text { direct two-photon } \\
\text { single ionization }\end{array}$ & $(3.0 \pm 1.2) \times 10^{-51}$ \\
\hline (4) & $\mathrm{He}$ & $(42.8 \pm 0.4)$ & $\begin{array}{l}\text { direct two-photon } \\
\text { double ionization }\end{array}$ & $(1.6 \pm 0.6) \times 10^{-52}$ \\
\hline
\end{tabular}

${ }^{\mathrm{a}}$ Valid for a photon pulse duration of $25 \mathrm{fs}$.

$$
\frac{d N_{+}}{d H_{p h}}=N \sigma_{\mathrm{I}}-N_{+} \sigma_{\mathrm{I}}-N_{+} \sigma_{\mathrm{II}} .
$$

The first and the third term on the right side of Eq. (3) describe generation and annihilation of a $\mathrm{Ne}^{+}$ion by the first and the second photon absorbed, respectively. The second term takes into consideration that the initial number of atomic targets $N$ may be considerably reduced by the first step photoionization within a single photon pulse $[12,16]$.

Equation (3) represents a differential equation with the solution

$$
N_{+}\left(H_{p h}\right)=N \frac{\sigma_{\mathrm{I}}}{\sigma_{\mathrm{I}}+\sigma_{\mathrm{II}}}\left\{1-\exp \left[-\left(\sigma_{\mathrm{I}}+\sigma_{\mathrm{II}}\right) H_{p h}\right]\right\} .
$$

Neglecting here the weak $\mathrm{Ne}^{3+}$ signal [Fig. 2(a)], one finally obtains for the $\mathrm{Ne}^{2+}$ to $\mathrm{Ne}^{+}$ion yield ratio

$$
\begin{gathered}
\frac{N_{2+}}{N_{+}}=\frac{N_{+}\left(\sigma_{\mathrm{II}}=0\right)-N_{+}}{N_{+}} \underset{H_{p h} \rightarrow 0}{\longrightarrow} \frac{\sigma_{\mathrm{II}}}{2} H_{p h}, \\
\underset{H_{p h} \rightarrow \infty}{\longrightarrow} \frac{\sigma_{\mathrm{II}}}{\sigma_{\mathrm{I}}} .
\end{gathered}
$$

Our corresponding experimental result, i.e., the $\mathrm{Ne}^{2+}$ to $\mathrm{Ne}^{+}$ ion yield ratio for sequential two-photon double ionization of neon at $42.8 \mathrm{eV}$ photon energy, is shown by curve A in Fig. 3 . For low photon exposure, the ratio increases linearly with $H_{p h}$, as predicted by Eq. (5). Also a deviation from linearity beyond $2 \times 10^{17}$ photons per $\mathrm{cm}^{2}$ is obvious which confirms our assumptions. In the saturation regime at high photon exposure, the $\mathrm{Ne}^{2+}$ to $\mathrm{Ne}^{+}$ratio converges to the constant $\sigma_{\text {II }}$ to $\sigma_{\mathrm{I}}$ cross-section ratio. With the known one-photon single ionization cross section of $\mathrm{Ne}, \sigma_{\mathrm{I}}=\sigma_{\mathrm{Ne} \rightarrow \mathrm{Ne}^{+}}^{(1)}=(8.2 \pm 0.3)$ $\times 10^{-18} \mathrm{~cm}^{2}$ [20], it allows the evaluation of the single photoionization cross section of $\mathrm{Ne}^{+}$to amount to $\sigma_{\text {II }}$ $=\sigma_{\mathrm{Ne}^{+} \rightarrow \mathrm{Ne}^{2+}}^{(1)}=(7.0 \pm 1.0) \times 10^{-18} \mathrm{~cm}^{2}$. The cross section for the entire sequential two-photon double ionization process [Fig. 1(d)] then may be approximated by $\sigma_{\mathrm{I}} \sigma_{\mathrm{II}} \frac{\Delta t(=25 \mathrm{fs})}{2}$ which results in an experimental value of $(7.2 \pm 2.5)$ $\times 10^{-49} \mathrm{~cm}^{4} \mathrm{~s}$ [Table I (1)], similar to recent results on molecular nitrogen [12] but by more than two orders of magnitude higher than for the direct two-photon process on $\mathrm{Ne}^{2+}$ discussed above [Table I (3)].
Obviously, sequential and direct two-photon processes differ significantly with respect to strength and photon intensity dependence (Fig. 3). The latter concerns, in particular, the relevant quantity to express the strength of the photon field. Direct processes depend on the (mean) irradiance $E$ $=H_{p h}(\hbar \omega / \Delta t)$ [Eq. (1)]. A sequence of one-photon processes via long-living ionic states, on the other hand, may be expressed as a function of photon exposure $H_{p h}$ [Eq. (4)] without explicit dependence on the pulse duration $\Delta t$ as long as $\Delta t$ is short enough so that intermediate targets may be regarded to be frozen, i.e., do not move out of the interaction zone during a single photon pulse.

In this context, we have studied $\mathrm{Ne}$ also just below its threshold for sequential two-photon double ionization via $\mathrm{Ne}^{+}$at $41.0 \mathrm{eV}$ [Fig. 1(d)] and changed the photon energy from 42.8 to $38.4 \mathrm{eV}$. As a result the $\mathrm{Ne}^{3+}$ signal, in fact, disappeared and the $\mathrm{Ne}^{2+}$ signal drastically decreased in the corresponding ion TOF spectrum shown in Fig. 2(c). The remaining $\mathrm{Ne}^{2+}$ signal, again, is not strongly affected by higher FEL harmonics and must be due to multiphoton processes because it also vanishes when moving the experimental setup out of focus [Fig. 2(d)].

One explanation for the $\mathrm{Ne}^{2+}$ signal in the ion TOF spectrum measured at $(38.4 \pm 0.4) \mathrm{eV}$ photon energy [Fig. 2(c)] might be the direct two-photon double ionization of the atom [Fig. 1(g)]. Such a process has recently been studied on helium at $41.8 \mathrm{eV}$ photon energy $[9,10]$. An alternative scheme would be the two-photon single ionization of $\mathrm{Ne}^{+}$as the intermediate target, i.e., in total a three-photon excitation [Fig. 1(b)] in analogy to the corresponding process on $\mathrm{Ne}^{2+}$ [Fig. 1(e)] as discussed above. The two excitation schemes may be distinguished by the different photon intensity dependences of the respective $\mathrm{Ne}^{2+}$ to $\mathrm{Ne}^{+}$ion yield ratios according to Eq. (1) [and in analogy to Eq. (2)]:

two-photon double ionization of $\mathrm{Ne}: \frac{N_{2+}^{(2)}}{N_{+}^{(1)}}=\frac{\sigma_{\mathrm{Ne} \rightarrow \mathrm{Ne}^{2+}}^{(2)}}{\sigma_{\mathrm{Ne} \rightarrow \mathrm{Ne}^{+}}^{(1)}} \frac{H_{p h}}{\Delta t}$,

two-photon single ionization of $\mathrm{Ne}^{+}: \frac{N_{2+}^{(2)}}{N_{+}}=\frac{\sigma_{\mathrm{Ne}^{+} \rightarrow \mathrm{Ne}^{2+}}^{(2)}}{2} \frac{H_{p h}^{2}}{\Delta t}$. 
Our corresponding experimental result for the $\mathrm{Ne}^{2+}$ to $\mathrm{Ne}^{+}$ ion yield ratio at $38.4 \mathrm{eV}$ photon energy is shown by curve $\mathrm{B}$ in Fig. 3 and does not show a linear [Eq. (6)] but a clear square dependence on $H_{p h}$ [Eq. (7)]. Fitting the data with the model curve of Eq. (7) yields a two-photon single ionization cross section for $\mathrm{Ne}^{+}$of $\sigma_{\mathrm{Ne}^{+} \rightarrow \mathrm{Ne}^{2+}}^{(2)}=(1.6 \pm 0.6) \times 10^{-50} \mathrm{~cm}^{4} \mathrm{~s}$ as a fit parameter [Table I (2)]. The value is more than five times higher than for the same type of process we have observed on $\mathrm{Ne}^{2+}$ [Table I (3)]. An explanation might be the existence of $\mathrm{Ne}^{+}: 2 p \rightarrow 4 s$ excited states at $38.2 \mathrm{eV}$ [21], i.e., very close to our photon energy of $(38.4 \pm 0.4) \mathrm{eV}$. As a consequence, the two-photon single ionization of $\mathrm{Ne}^{+}$would be rather a sequential process via a real resonance state [Fig. 1(c)] than a direct one via a virtual state [Fig. 1(b)].

In Table I, our cross-section values for the different twophoton excitations we have investigated are summarized. They express the strength and the role of an ionization process, respectively. The given uncertainties arise mainly from signal background and statistics and the determination of FEL pulse energy, spot size, and pulse duration. For data evaluation based on Eq. (1), we have assumed a rectangular temporal distribution of the photon pulses since the real one was not known. However, in the case of a Gaussian temporal distribution, the cross-section data in lines (2)-(4) of Table I have to be multiplied by a factor of about 1.5 . In order to validate our data, we have also repeated measurements on direct two-photon double ionization of helium $(\mathrm{He})$, the only element where direct multiphoton ionization in the soft-x-ray regime has been observed so far $[9,10]$. Fitting experimental data for the $\mathrm{He}^{2+}$ to $\mathrm{He}^{+}$ion yield ratio, measured at a photon energy of $42.8 \mathrm{eV}$, by a model curve analogous to Eq. (6) (curve D in Fig. 3) and using the known one-photon single ionization cross section of $\mathrm{He}, \sigma_{\mathrm{He} \rightarrow \mathrm{He}^{+}}^{(1)}=(2.75 \pm 0.08)$ $\times 10^{-18} \mathrm{~cm}^{2}$ [20], we have carried out a cross section of $\sigma_{\mathrm{He} \rightarrow \mathrm{He}^{2+}}^{(2)}=(1.6 \pm 0.6) \times 10^{-52} \mathrm{~cm}^{4} \mathrm{~s}$ [Table I (4)]. The value agrees fairly well with the estimation of $1 \times 10^{-52} \mathrm{~cm}^{4} \mathrm{~s}$ reported in Ref. [10] for a photon energy of $41.8 \mathrm{eV}$ and a recent theoretical prediction of $4 \times 10^{-52} \mathrm{~cm}^{4} \mathrm{~s}$ at $43 \mathrm{eV}$ [22].

In summary, we have presented a comprehensive and quantitative study of multiphoton ionization in the xuv regime. Our measurements were performed on neon and helium atoms in a focused beam at the new free-electron laser FLASH facility in Hamburg by means of ion time-of-flight mass-to-charge spectroscopy. Variation and determination of photon exposure, irradiance, and photon energy has allowed us to distinguish and compare different excitation schemes and to derive corresponding atomic and ionic photoionization cross sections. The dominant role of sequential multiphoton processes via ionic and resonance states compared to direct multiphoton excitation via virtual states is clearly demonstrated.

We thank the FLASH team for the very successful operation of the FEL and the optimal beamtime conditions; we also thank U. Jastrow, U. Kroth, H. Schöppe, J. R. Schneider, B. Sonntag, and G. Ulm for continuous support.
[1] P. G. O'Shea and H. P. Freund, Science 292, 1853 (2001).

[2] H. C. Kapteyn and T. Ditmire, Nature (London) 420, 467 (2002).

[3] V. Ayvazyan et al., Phys. Rev. Lett. 88, 104802 (2002).

[4] V. Ayvazyan et al., Eur. Phys. J. D 37, 297 (2006).

[5] T. Sekikawa, A. Kosuge, T. Kanai, and S. Watanabe, Nature (London) 432, 605 (2004).

[6] J. Feldhaus, J. Arthur, and J. B. Hastings, J. Phys. B 38, S799 (2005).

[7] H. Wabnitz et al., Nature (London) 420, 482 (2002).

[8] N. Miyamoto M. Kamei, D. Yoshitomi, T. Kanai, T. Sekikawa, T. Nakajima, and S. Watanabe, Phys. Rev. Lett. 93, 083903 (2004)

[9] Y. Nabekawa, H. Hasegawa, E. J. Takahashi, and K. Midorikawa, Phys. Rev. Lett. 94, 043001 (2005).

[10] H. Hasegawa, E. J. Takahashi, Y. Nabekawa, K. L. Ishikawa, and K. Midorikawa, Phys. Rev. A 71, 023407 (2005).

[11] H. Wabnitz et al., Phys. Rev. Lett. 94, 023001 (2005).
[12] A. A. Sorokin, S. V. Bobashev, K. Tiedtke, and M. Richter, J. Phys. B 39, L299 (2006).

[13] E. P. Benis, D. Charalambidis, T. N. Kitsopoulos, G. D. Tsakiris, and P. Tzallas, Phys. Rev. A 74, 051402(R) (2006).

[14] D. C. Morton, Astrophys. J., Suppl. Ser. 149, 205 (2003).

[15] M. Richter et al., Appl. Phys. Lett. 83, 2970 (2003).

[16] A. A. Sorokin et al., Appl. Phys. Lett. 89, 221114 (2006).

[17] M. V. Yurkov (private communication).

[18] S. Düsterer et al., Opt. Lett. 31, 1750 (2006).

[19] M. Protopapas, C. H. Keitel, and P. L. Knight, Rep. Prog. Phys. 60, 389 (1997).

[20] J. M. Bizau and F. J. Wuilleumier, J. Electron Spectrosc. Relat. Phenom. 71, 205 (1995).

[21] A. E. Kramida, C. M. Brown, U. Feldman, and J. Reader, Phys. Scr. 74, 156 (2006).

[22] E. Foumouo, G. L. Kamta, G. Edah, and B. Piraux, Phys. Rev. A 74, 063409 (2006). 